\title{
Sol-Gel Method Applied to Crystalline Materials
}

\author{
Alessandro Dell'Era ${ }^{1, *(1)}$ and Michelina Catauro ${ }^{2}$ (D) \\ 1 Department of Basic and Applied Sciences for Engineering, Sapienza University of Rome, \\ Via Antonio Scarpa 16, 00161 Rome, Italy \\ 2 Department of Engineering, University of Campania "Luigi Vanvitelli", Via Roma 29, I-81031 Aversa, Italy; \\ michelina.catauro@unicampania.it \\ * Correspondence: alessandro.dellera@uniroma1.it
}

Citation: Dell'Era, A.; Catauro, M. Sol-Gel Method Applied to

Crystalline Materials. Crystals 2021, 11,903. https://doi.org/10.3390/ cryst11080903

Received: 27 July 2021

Accepted: 30 July 2021

Published: 31 July 2021

Publisher's Note: MDPI stays neutral with regard to jurisdictional claims in published maps and institutional affiliations.

Copyright: (c) 2021 by the authors. Licensee MDPI, Basel, Switzerland. This article is an open access article distributed under the terms and conditions of the Creative Commons Attribution (CC BY) license (https:// creativecommons.org/licenses/by/ $4.0 /)$.
Keywords: biomaterials; nanoparticles; thin films and coatings; organic-inorganic hybrid materials; catalysts; sensors and biosensors; energy; optics

Sol-gel chemistry is a versatile synthesis used to produce modern materials at nearroom temperature. Glasses, ceramics, composites, and new hybrid materials that are not easy to obtain using other methods have been, instead, obtained in the last three decades and nowadays are widely used. Changing the chemical composition, many parameters of the sol-gel process can be adapted to control the properties and the microstructure of the obtained materials. Sol-gel technology is a multidisciplinary science which allows the expansion of materials for many applications. In this Special Issue, special attention is paid to the properties of materials obtained by using sol-gel methods and to their potential applications in environmental science and materials science as in catalysis, optics, electronics, energy, biosensors, medicine, and so on. This issue collects four contributions, starting with the paper of A.I.M. Greer et co-workers [1] that studied $\mathrm{TiO}_{2}$ in the anatase polymorph synthesized by sol-gel method as an effective self-cleaning agent under subaquatic conditions. It is known that $\mathrm{TiO}_{2}$ in the anatase polymorph is a semi-conductor with a band gap energy of $3.2 \mathrm{eV}$, able to degrade pollutants in air. Positive charges have an oxidizing action on any organic matter (dirt) on the surface, and negative charges combine with atmospheric oxygen to create oxygen radicals that react with oxidized dirt to produce a release of $\mathrm{CO}_{2}$ and $\mathrm{H}_{2} \mathrm{O}$ and ultimately a cleaning of $\mathrm{TiO}_{2}$ surface. Due to the increasing range of subaquatic system monitoring devices based on optics properties, A.I.M. Greer et co-workers have also tested $\mathrm{TiO}_{2}$ self-cleaning capacity under water by using crude oil from North Sea Troll B. Dopants of higher (Mn), lower (Gd), and equal (Si) oxidation states were all tested as well as the effects of both the increase in the anatase particle size and the surface roughness. They established that the undoped $\mathrm{TiO}_{2}$ has the best performances and that the larger the particle size, the lower the roughness and the lower the performances. Finally, they demonstrate that sol-gel-derived $\mathrm{TiO}_{2}$ in the anatase polymorph is an effective self-cleaning coating under subaquatic conditions. In addition, biological contamination may also be cleansed from the surface using the titania coating, making it suitable for a range of subaquatic applications, where oil or biofilms are known contaminants. In a different context, A. Alsaad and co-workers [2] studied the properties of $\mathrm{TiO}_{2}$ nanoparticles prepared by using the sol-gel method. They report the synthesis of hybrid thin films based on poly(methylmethacrylate) (PMMA) and poly(vinylalcohol) (PVA), doped with different concentrations of titanium dioxide nanoparticles $\left(\mathrm{TiO}_{2} \mathrm{NPs}\right)$, which obtained a hybrid polymeric nanocomposite thin films. This kind of polymeric nanocomposite thin films has received great attention, owing to their unusual physical, chemical, and optical properties. Indeed, high refractive index polymers are good candidates for advanced display devices, micro lens components, organic light emitting diode devices, and lithography. Synergy between metal nanoparticles and polymers exalts the optical properties and modifies the mechanical behavior of the polymer composite. The advancement of polymeric composite 
thin films is basically determined by the selection of ionic fillers and optimum filler loads. In this scenario, A. Alsaad and co-workers studied the transmittance, reflectance, absorption coefficient, optical constants, and optical dielectric functions of hybrid polymeric thin films. Increasing $\mathrm{TiO}_{2}-\mathrm{NPs}$ contents in PMMA-PVA polymeric thin films lead to the increase in refractive index values, while the optical band gap energy $(4.101 \mathrm{eV})$ slightly decreases as $\left(\mathrm{TiO}_{2}-\mathrm{NPs}\right)$ doping levels introduced in (PMMA-PVA) polymeric thin films are increased. The obtained high refractive indices of these thin films indicate that they could be key candidates for strong optical confinement applications and can enhance the optical intensities of nonlinear interactions in optical components of modern devices. The high transmittance, wide bandgap energy, and high refractive index indicate that these films could be employed in the fabrication of a wide range of optoelectronic devices. As far as the sensors field is concerned, D. Triyono and co-workers [3] studied the effect of $\mathrm{Zr}$ as a doping agent into $\mathrm{LaFeO}_{3}$. The interest in $\mathrm{LaFeO}_{3}$ among perovskite inorganic materials significantly increased due to their applicability as chemical sensors and electrode materials, etc. Doping the La-site with divalent metals and/or Fe-site with transition metals has been reported to increase the structural and thermal stability, magnetic, and electrical properties as compared with the parent compound.

Many papers on $\mathrm{LaFeO}_{3}$ perovskite doping by substitution of La-site with divalent metals and/or Fe-site with transition metals have been produced, but research investigations on the effect of $\mathrm{Zr}$ cations on the Fe-site of $\mathrm{LaFeO}_{3}$ are still lacking information. The question of the effect of $\mathrm{Zr}$, as tetravalent cations occupying the Fe-site of $\mathrm{LaFeO}_{3}$ on the structural parameters and electrical transport mechanism, has not been deeply studied yet. In this context, the authors studied and characterized $\mathrm{LaFe}_{(1-\mathrm{x})} \mathrm{Zr}_{\mathrm{x}} \mathrm{O}_{3}$ by XRD, Raman scattering analysis, SEM, and impedance spectroscopy method. The $\mathrm{LaFe}_{(1-\mathrm{x})} \mathrm{Zr}_{\mathrm{x}} \mathrm{O}_{3}(\mathrm{x}=0.01$, $0.05)$ ceramics were prepared by sol-gel and annealing methods. Their analysis revealed that the crystal structure tends to preserve its ideal orthorhombic structure, following the increase in driving force of the $\mathrm{Fe} / \mathrm{ZrO}$ octahedral tilting. The frequency-dependent dielectric parameters at each temperature decreased with increasing $\mathrm{Zr}$ content, while the temperature dependence with dielectric relaxation increased with increasing $\mathrm{Zr}$ content. $\mathrm{Zr}$ substitution, until $5 \%$ molar concentration, still preserves the single phase of orthorhombic perovskite structure with Pbnm symmetry, followed by the increase in lattice constant with increasing $\mathrm{Zr}$ content. Analyses revealed a decrease in lattice disorder and crystal distortion with $\mathrm{Zr}$ content which is consistent with the decreased calculated tolerance factor. However, the average crystallite and grain size decrease with $\mathrm{Zr}$ content indicating the reduced degree of crystallinity. Finally, the dielectric parameters decrease with increasing $\mathrm{Zr}$ content and the activation energy calculated in the relaxation and dc conduction mechanism is found increase with increasing $\mathrm{Zr}$ content. The last paper deals with bioactive material synthesized by sol-gel method. T.H. Dang and and co-workers [4] produced a bioactive glass with the composition of $70 \mathrm{SiO}_{2}-30 \mathrm{CaO}$ (mol.\%) by using, in particular, a modified sol-gel process in hot water without acid catalysts. The original bioactive glass was first discovered in 1970 by Larry L. Hench. The bioactivity of this glass is expressed by the formation of a new layer of hydroxyapatite $\mathrm{Ca}_{10}\left(\mathrm{PO}_{4}\right)_{6}(\mathrm{OH})_{2}(\mathrm{HA})$ on its surface when immersed in a physiological solution or implanted in the human body (SBF). Many bioactive glasses have then been synthesized and used as artificial biomaterials for bone substitutes. In the past fifty years, melting and sol-gel have been the two main methods used for the synthesis of bioactive glasses. However, melting bioactive glasses require synthesis processes at high temperatures, where volatile compounds, such as $\mathrm{P}_{2} \mathrm{O}_{5}$, can be evaporated, resulting in a difference in glassy compositions. Furthermore, the obtained glasses usually have dense structures and low specific surface area values, which produce low bioactivities of synthetic glasses. The sol-gel method overcomes the drawbacks of the melting technique because glasses are synthesized at lower temperatures. Sol-gel bioactive glasses normally have mesoporous structures with larger specific surface area values, leading to higher bioactivity. However, most sol-gel bioactive glasses are prepared using strong inorganic acids as catalysts, which have negative effects on health and the environment 
in view of green chemistry. In this context, the breakthrough is to obtain a glass as a totally amorphous material with a mesoporous structure without using acid catalysts. The in vitro experiments confirmed the bioactivity of glass by formation of apatite after one day. In vitro assay in cellular medium confirmed good biocompatibility of synthetic glass. Therefore, the bioactive glass synthesized by an environmentally friendly method in this study can find potential application as artificial bone substitutes.

In summary, this Special Issue highlights the importance of the sol-gel method applied to crystalline materials which are useful in a wide range of applications, reaching a big breakthrough in many fields of science. We would like to thank all authors who have contributed their brilliant papers to this Special Issue, and we also wish to thank the Editorial Office of Crystals for the fast and professional handling of the manuscripts during the whole submission process.

\section{References}

1. Greer, A.I.M.; Moodie, D.; Kerr, G.; Gadegaard, N. Sol-Gel Coatings for Subaquatic Self-Cleaning Windows. Crystals 2020, 10, 375. [CrossRef]

2. Alsaad, A.; Al Dairy, A.R.; Ahmad, A.; Qattan, I.A.; Al Fawares, S.; Al-Bataineh, Q. Synthesis and Characterization of Polymeric (PMMA-PVA) Hybrid Thin Films Doped with TiO2 Nanoparticles Using Dip-Coating Technique. Crystals 2021, 11, 99. [CrossRef]

3. Triyono, D.; Purnamasari, I.; Rafsanjani, R.A. Effect of the Zr-Substitution on the Structural and Electrical Properties of LaFeO3: XRD, Raman Scattering, SEM, and Impedance Spectroscopy Study. Crystals 2020, 10, 399. [CrossRef]

4. Dang, T.H.; Bui, T.H.; Guseva, E.V.; Ta, A.T.; Nguyen, A.T.; Hoang, T.T.H.; Bui, X.V. Characterization of Bioactive Glass Synthesized by Sol-Gel Process in Hot Water. Crystals 2020, 10, 529. [CrossRef] 